\title{
Evaluation of a Commercial Telehealth Weight Loss and Management Program
}

\author{
Win Guan $^{1 *}$, Andrew Thaw ${ }^{2}$, Sabrina N. Grondhuis ${ }^{2}$ and Andi Schaechter ${ }^{1}$ \\ ${ }^{1}$ Louisiana Department of Health, USA \\ ${ }^{2}$ Department of Psychology and Neuroscience, Millsaps College, USA
}

"Corresponding author: Win Guan, Louisiana Department of Health, USA, Tel: +5049823237; E-mail: wguan22@gmail.com

Received date: October 11, 2018; Accepted date: November 08, 2018; Published date: November 16, 2018

Copyright: @ 2018 Win Guan, et al., This is an open-access article distributed under the terms of the Creative Commons Attribution License, which permits unrestricted use, distribution, and reproduction in any medium, provided the original author and source are credited.

\begin{abstract}
Objective: This study assesses the effectiveness of the Bright Line Eating Boot Camp (BLE: BC), an eight-week telehealth weight management program.

Methods: Data come from participants in the BLE: BC research program. The final sample $(n=5,374)$ contained primarily white adults $(92.8 \%)$, females $(95.2 \%)$, and individuals who reported high socioeconomic status $(96.0 \%$ had completed at least some college and $47.4 \%$ reported an annual family income of at least $\$ 100,000$ ). We focus in this manuscript on the primary outcomes of percent weight loss and change in body mass index from baseline. Secondary outcomes include program satisfaction and perceptions of healthy eating.

Results: Approximately $95 \%$ of participants lost weight between baseline and completion of the BLE: BC. During the eight weeks, average percent weight loss was $7.8(\mathrm{SD}=7.5)$ and body mass index declined by an average of 2.6 $(S D=2.3)$. Spearman's correlation tests show that participants who reported expending more effort and participating more in the program reported greater percent weight loss ( $r s=0.39, p<0.001$ and $r s=0.34, p<0.001$, respectively) and larger reduction in body mass index $(r s=0.36, p<0.001$ and $r s=0.33, p<0.001$, respectively). The majority of participants $(88.6 \%)$ reported being satisfied with the program and $90.4 \%$ reported that healthy eating became easier.

Conclusion: The results of this study support the efficacy of the BLE: BC as a fully online, telehealth weight loss program. Future studies should assess the long-term weight loss and maintenance of BLE: BC participants and endeavor to specify mechanisms for the observed weight change. Although we acknowledge limitations in generalizability of the results due to the lack of a comparison group and selection bias in the sample, the results show clinically-significant weight loss among the majority of BLE: BC participants.
\end{abstract}

Keywords: Obesity; Body weight; Overweight; Weight loss; Body mass index; Weight management; Weight reduction programs; Obesity management; Health promotion

\section{Introduction}

The 2017 National Health and Nutrition Examination Survey (NHANES) estimates that $39.8 \%$ of American adults suffer from obesity $(\mathrm{BMI} \geq 30$ ). The US obesity epidemic presents significant public health concerns, including increased risk of such comorbid conditions as type 2 diabetes, hypertension, heart disease [1], and increased mortality risk [2]. Obesity also carries an economic burden that stems from such correlates as increased healthcare costs $(\$ 210$ billion per year) [3], job absenteeism ( $\$ 4.3$ billion per year) [4], and lower work productivity ( $\$ 506$ per worker with obesity) [5]. The etiology of obesity includes modifiable risk factors particularly related to physical activity and nutrition that clinical weight loss interventions and commercial weight loss programs historically target. Although the prevalence of telehealth (web-based) programs has increased in recent years, studies evaluating the efficacy of telehealth programs have proven largely inconclusive, in part because of the wide variation in program design and intensity [6]. In an initial systematic review of outcome evaluations of commercial weight loss programs, Tsai and
Wadden [7] found only one randomized control study and no case series: Results of this evaluation of the eDiets.com program [8] showed a decrease of $0.9 \% \mathrm{WL}$ at 16 weeks and $1.1 \% \mathrm{WL}$ at 1 year. They did not examine BMI changes.

Since the review of Neve et al. [6], the popularity of web-based programs has grown dramatically. As a result, Gudzune et al. [9] conducted an updated review. They included only randomized control trials and added evaluations of two web-based programs. They found an approximate 1.2 decrease in BMI among participants of the Lose It! application at 6-month follow-up [10]; they did not examine percent WL. Twelve-week results for participants in (a) basic and (b) enhanced versions of the Biggest Loser Club weight loss program showed a 2.3 $\% \mathrm{WL}$ and $3.3 \% \mathrm{WL}$, respectively, and 0.7 and 1.0 decreases in BMI [11]. We see several implications of these systematic reviews of commercial weight loss programs. First, it is noteworthy that commercial telehealth programs seldom publish weight loss outcomes. This keeps both medical providers and the general public in the dark about their efficacy. Second, researchers should not neglect case series evaluations of web-based programs. Although randomized control trials remain the gold-standard, case series evaluations can provide important descriptive information. Finally, the studies of eDiets.com, the Lose It! app, and the Biggest Loser Club that were included in the 
reviews show modest weight loss results, leaving open the question of whether commercial web-based weight loss programs can produce significant weight loss among individuals with obesity.

As a result, this study addresses the limitations of previous research by employing a prospective case series design to evaluate the primary outcomes of the Bright Line Eating: Boot Camp (BLE: BC) program, an eight-week commercial telehealth weight loss program. We describe change in weight and body mass index (BMI) from baseline to completion of the 8-week BLE: BC. Two factors distinguish the BLE: BC program: (1) The delivery system relies entirely on a telehealth model and (2) the intervention is grounded in the processed food addiction framework. The following sections describe the theoretical foundation and structure of the BLE: BC program.

\section{The processed food addiction framework}

The processed food addiction paradigm [12] hypothesizes that certain foods--particularly palatable foods--can create neurobiological and behavioral responses that resemble drug addiction. Recent research has shown that eating highly-desired foods can exert effects on the brain that resemble those of certain addictive substances. Essentially, food intake stimulates reward mechanisms resulting in down regulation of dopamine D2 receptors (DD2R) [13-15]. This dopamine down regulation may lead individuals to consume larger and larger quantities of food, similar to the way in which drug use increases with repeated use and increased drug tolerance. Proponents of the model argue that the resulting pattern fuels obesity. In a systematic review of 52 studies on food addiction (22 on human subjects and 30 on animal subjects), researchers found significant support for the food addiction paradigm [16]. The review showed evidence for each of the key diagnostic constructs examined including neurological changes, preoccupation with the substance, impaired control, social impairments, risky use, tolerance/withdrawal, chronicity of the condition, and relapse.

\section{The Bright Line Eating Boot Camp Program}

The initial communication from BLE to prospective enrollees includes a three-part video series that describes (a) common mistakes that individuals make when they attempt to lose weight, (b) the regulation of hunger and appetite by the central nervous system, and (c) the science of willpower. Prospective enrollees can then decide whether to pay the participation fee to enroll in a BLE: BC. In 2015 and 2016, BLE offered the BC program three to five times per year, using a cohort-based system in which a group of individuals enrolled in the program and then moved through the 8 -week sequence together. Beginning in 2017, BLE offered the BC continuously, through a rolling enrollment. BLE: BC provides weekly online video modules, extra bonus modules from ancillary experts, vlogs (video blogs), and group coaching calls. To simulate the supportive environment found in 12step programs, BLE provides closed Facebook groups and also encourages participants to create their own support groups, such as small group meetings (masterminds) and one-one-one calls with a "buddy." In addition, BLE: BC encourages participants to use such other tools as daily accountability logs, diaries, food journals, meditation, and gratitude journals.

After registering for the program, participants move through an orientation period that includes a welcome video and a getting-started checklist with several videos and downloadable documents that explain the program in detail. Following this introduction, participants can access program components through the BLE: BC website. That website offers educational and motivational pre-recorded video modules that explain various features of the BLE program and also provide scientifically-based information about the psychology of weight loss, the neuroscience of eating behaviors, and the regulation of body weight. BLE releases a new module to BC participants each week that encourages specific behavioral changes, with explanations detailing why these changes prove important. The program includes a total of 127 videos of varying lengths (averaging about seven minutes), divided evenly across the eight weekly modules (resulting in 14-20 videos per module). Participants access the videos at their own pace.

Because these weekly modules detail the specific steps of the program and address common concerns or questions, these modules constitute the core of the BLE: BC. In addition to watching these educational videos, participants follow a food plan whose design aims to address overeating of foods that not only contain a high number of calories but also have been associated with abnormal physiological changes and excess intake [17-20]. Specifically, BLE identifies four "Bright Lines" that participants should avoid crossing. The structure of the Bright Lines, which allows participants to navigate their food choices successfully, includes:

- Abstaining from added sugars, including natural (such as maple syrup) and artificial (e.g., sucralose) sugars; this includes abstaining from processed foods that contain added sugar, such as certain types of peanut butter and ketchup. The rationale for avoiding not only natural but also artificial sweeteners rests on the premise that these isolated sweet substances promote excess food consumption and weight gain [18]. The program does allow sugars found in whole foods (such as fruit).

- Eliminating processed flour of any kind (including whole grain flour), because it is viewed in the program as a dose-dependent toxin.

- Eating three meals each day, with no snacks, which limits engagement with food and promotes "automatic" food behaviors. It also limits the feeding window, which has been shown to result in weight loss independent of food choice or caloric intake.

- Limiting the quantity of food consumed in each meal. Instead of counting calories, participants weigh their food as the primary way to moderate calories. Both the limitation of food quantity and the three-meal structure counteract the documented tendency of some individuals to underestimate sometimes drastically the number of calories they consume in a day [21-22].

BLE encourages participants to abstain from processed foods because of their elevated palatability and increased speed of absorption. In lieu of these foods, BLE urges participants to eat large quantities of vegetables with a modest amount of proteins, fruits, fats, and other foods. That structure allows participants to consume large quantities of food (especially low-calorie vegetables) without excessive caloric intake. Although the categories of foods for each meal remain the same from day to day, the program requires few limitations on the types of foods participants can choose in each category (e.g., proteins, fruits, fats, vegetables, and grains). The BLE program offers flexibility by offering alternative suggestions for different genders, for individuals with specific dietary preferences (such as vegetarian or vegan), for individuals with specific health issues, and for different levels of energy expenditure.

One of the key features of the BLE: BC is the social support that it provides. Once participants enroll in the program, they are directed to 
a web page that assigns them to a private online support group of fellow participants, former participants, and BLE staff members. Participants within these groups can share their experiences openly, ask and answer questions, discuss difficulties, and share tips. Each group includes two paid moderators who answer specific questions about the program and ensure that discussions remain positive and helpful. These moderators are trained BLE staff members who are also former program participants. To augment the supportive environment, each group includes an additional 10-15 BLE: BC participants who have been successful in the program previously and have been invited to repeat the BLE: BC at no cost. The BLE staff encourages participants to share their daily menus, celebrate successes, and ask for assistance when they struggle with the program.

BLE: BC participants can also access a two-hour (live) questionand-answer call with senior BLE coaches each week. Participants may submit questions or comments to the coaches before the call. Because BLE records these calls and posts them on the BLE: BC website, participants who were unable to participate in the calls live can still access them. Participants may also sign up for a "buddy system," in which fellow participants contact each other daily to provide support. Alternatively, they may opt to join a mastermind group in which four participants meet in a 90-minute, structured weekly conference call to support each other. Finally, at the end of the Boot Camp, BLE offers participants a webinar that outlines how to continue with the Bright Line Eating program after they matriculate from the Boot Camp. All programmatic resources remain available to registered users ad libitum, allowing them to return to the modules, weekly calls, and program information at any time, indefinitely.

\section{Participants}

Participants in this study come from the BLE: BC Research Program. The BLE: BC Research Program is a convenient sample of BLE: BC participants who agreed to complete a series of surveys for the evaluation of the program. Informed consent was gathered from each of these participants. During this process, participants were provided a detail protocol for the use, security, and management of the survey data.

From October, 2014 until March, 2018, 18,778 individuals enrolled in a BLE: BC. Of those, 12,040 elected to participate in the research program. BLE asks research program participants to complete: (1) a baseline survey, (2) eight weekly surveys, and (3) an ending survey. The baseline survey was distributed to participants following orientation for the BLE: BC program where participants were introduced to the social support groups such as private online support groups. Topics in the baseline survey included socio-demographic data, prior experience with other weight loss programs, motivation for completing the BLE: BC program, weight status, health status, and psychosocial measures. Weekly surveys are completed at the end of each of the 8 weeks in the BLE: BC program. Following completion of the program, participants go through an exit process within which one of the components is completing the BLE: BC ending survey. In general, topics in the ending survey parallel that of the baseline survey in order to measure change from beginning to the end of the BLE: BC program.

The current study uses data from the baseline and ending surveys. As of March, 2018, 9,996 participants had completed the baseline survey and 6,494 had completed the ending survey, with a total of
5,877 participants completing both the baseline and ending surveys. We removed from the analyses participants with missing data for the primary and secondary outcomes defined below, resulting in a final sample of 5,374 participants.

\section{Measures}

Weight loss and BMI change served as the primary outcome measures. We measured weight loss as percent weight loss (\%WL), calculated as the percentage change in self-reported weight between the baseline and ending survey. We used participants' self-reported height and weight in the standard BMI formula $(\mathrm{kg} / \mathrm{m} 2)$ to calculate BMI.

Secondary outcome variables included program satisfaction and perceived ease of healthy eating. To measure program satisfaction, we used participant's responses to the question, "Overall, how satisfied were you with your BLE: BC experience," which included a 6-point Likert scale that ranged from "very dissatisfied" to "very satisfied." To tap perceived ease of healthy eating, we used responses to the question, "As a result of the BLE program, does it feel like healthy eating has gotten easier, harder, or stayed the same?" This question also included a 6-point Likert scale.

We stratified results for the primary and secondary outcomes by baseline weight status and respondents' self-reported adherence to BLE: BC program activities. To measure baseline weight status, we used BMI categories of normal weight (BMI of 18.5-25), overweight (BMI of 25-30), class 1 obesity (BMI of 30-35), class 2 obesity (BMI of 35-40), and class 3 obesity (BMI of 40+). To measure adherence to the BLE: BC program, we used two ending survey questions that tapped level of effort and level of participation.

\section{Analytic strategy}

We used STATA SE 12.0 to analyze the data. We report descriptive statistics for primary outcomes, secondary outcomes, and covariates. To examine sex differences in all measures, we employed t-tests. To examine associations between baseline weight status and (a) weight loss (\%WL and BMI change) and (b) program adherence (effort and participation), we used Spearman correlation tests. We also used t-test comparisons of (a) program satisfaction and (b) perceptions of the ease of healthy eating to clinically-significant weight loss ( $\geq 5 \% \mathrm{WL})$.

\section{Results}

\section{Descriptive statistics}

(Table 1) presents descriptive statistics of the final sample $(n=5,374)$. Means and proportions appear (a) for the total sample and (b) by sex. The final sample was predominantly female (95.2\%) and white $(92.8 \%)$, with an average age of 55.0 (range 18-86). Participants also reported high socioeconomic status $(96.0 \%$ reported completing at least some college and $47.4 \%$ reported family income above $\$ 100,000)$. At baseline, $12.6 \%$ of participants lay within the normal BMI range, $30.9 \%$ were overweight, $26.4 \%$ reported class 1 obesity, $16.0 \%$ reported class 2 obesity, and $14.1 \%$ reported class 3 obesity. Females were significantly more likely to report normal weight at baseline, while males were more likely to be severely (class 3 ) obese at baseline. 
Citation: Guan W, Thaw A, Grondhuis S, Schaechter A (2018) Evaluation of a Commercial Telehealth Weight Loss and Management Program. J Nutr Weight Loss 3: 1000114.

Page 4 of 8

\begin{tabular}{|c|c|c|c|c|c|c|}
\hline \multicolumn{7}{|c|}{$\begin{array}{l}\text { Total Male Female } \\
(n=5,374)(n=259)(n=5,115)\end{array}$} \\
\hline \multicolumn{7}{|c|}{ Mean (\%) SD Mean (\%) SD Mean (\%) SD } \\
\hline Age & 55 & 10.8 & 57.3 & 12.7 & 54.9 & 10.7 \\
\hline Race (White) & $92.80 \%$ & -- & $93.60 \%$ & -- & $92.80 \%$ & -- \\
\hline Some college or more & $96.00 \%$ & -- & $95.00 \%$ & -- & $96.00 \%$ & -- \\
\hline Income $>\$ 100,000^{\star *}$ & $47.40 \%$ & -- & $54.60 \%$ & -- & $47.10 \%$ & -- \\
\hline \multicolumn{7}{|l|}{ Level of Effort } \\
\hline No effort at all & $0.45 \%$ & -- & $0.78 \%$ & -- & $0.43 \%$ & -- \\
\hline Some effort ${ }^{\star *}$ & $8.60 \%$ & -- & $12.90 \%$ & -- & $8.40 \%$ & -- \\
\hline A lot of effort & $35.00 \%$ & -- & $35.90 \%$ & -- & $34.90 \%$ & -- \\
\hline Gave it my all ${ }^{* *}$ & $56.00 \%$ & -- & $50.40 \%$ & -- & $56.30 \%$ & -- \\
\hline \multicolumn{7}{|l|}{ Level of Participation } \\
\hline Hardly participated & $2.70 \%$ & -- & $2.70 \%$ & -- & $2.70 \%$ & -- \\
\hline Participated some ${ }^{* *}$ & $8.60 \%$ & -- & $12.80 \%$ & -- & $8.40 \%$ & -- \\
\hline Participated a lot & $24.30 \%$ & -- & $24.00 \%$ & -- & $24.30 \%$ & -- \\
\hline From beginning ${ }^{* *}$ & $64.50 \%$ & -- & $60.50 \%$ & -- & $64.70 \%$ & -- \\
\hline \multicolumn{7}{|l|}{ Baseline weight status } \\
\hline Normal weight ${ }^{\star * *}$ & $12.60 \%$ & -- & $6.60 \%$ & -- & $12.90 \%$ & -- \\
\hline Overweight & $30.90 \%$ & -- & $29.70 \%$ & -- & $30.90 \%$ & -- \\
\hline Class 1 obesity** & $26.40 \%$ & -- & $29.00 \%$ & -- & $26.30 \%$ & -- \\
\hline Class 2 obesity & $16.00 \%$ & -- & $16.20 \%$ & -- & $16.00 \%$ & -- \\
\hline Class 3 obesity ${ }^{* * *}$ & $14.10 \%$ & -- & $18.50 \%$ & -- & $13.90 \%$ & -- \\
\hline Baseline weight $(\mathrm{kg})^{\star \star \star}$ & 88.3 & 20.6 & 103.4 & 25.8 & 87.5 & 20 \\
\hline End weight $(\mathrm{kg})^{\star \star \star}$ & 81.2 & 19 & 94.1 & 23.3 & 80.5 & 18.5 \\
\hline$\% \mathrm{WL}^{*}$ & 7.8 & 7.5 & 8.8 & 4.6 & 7.7 & 0.08 \\
\hline Baseline $B M I^{* \star *}$ & 32.3 & 7.1 & 33.8 & 7.5 & 32.3 & 7.1 \\
\hline End BMI** & 29.7 & 6.6 & 30.8 & 6.8 & 29.7 & 6.6 \\
\hline Change in $\mathrm{BMI}^{* *}$ & -2.6 & 2.3 & -3 & 1.8 & -2.6 & 2.4 \\
\hline Percent lost weight & $94.70 \%$ & -- & $94.60 \%$ & -- & $94.70 \%$ & -- \\
\hline Percent with $>5 \% \mathrm{WL}$ & $79.20 \%$ & -- & $82.30 \%$ & -- & $79.10 \%$ & -- \\
\hline \multicolumn{7}{|l|}{ Program satisfaction } \\
\hline Very dissatisfied & $0.70 \%$ & -- & $0.80 \%$ & -- & $0.60 \%$ & -- \\
\hline Dissatisfied & $0.90 \%$ & -- & $0.00 \%$ & -- & $1.00 \%$ & -- \\
\hline Neutral & $9.90 \%$ & -- & $9.60 \%$ & -- & $9.90 \%$ & - \\
\hline Satisfied & $17.10 \%$ & -- & $20.10 \%$ & -- & $17.00 \%$ & - \\
\hline Very satisfied & $71.50 \%$ & -- & $69.50 \%$ & -- & $71.60 \%$ & - \\
\hline
\end{tabular}


Citation: Guan W, Thaw A, Grondhuis S, Schaechter A (2018) Evaluation of a Commercial Telehealth Weight Loss and Management Program. J Nutr Weight Loss 3: 1000114.

Page 5 of 8

\begin{tabular}{|l|l|l|l|l|l|l|}
\hline \multicolumn{2}{|l|}{ Perception of healthy eating } & & & & \\
\hline Became easier & $90.40 \%$ & - & $90.90 \%$ & - & $90.40 \%$ & - \\
\hline No change & $1.90 \%$ & -- & $1.60 \%$ & -- & $1.90 \%$ & - \\
\hline Became harder & $7.70 \%$ & -- & $7.50 \%$ & -- & $7.70 \%$ & - \\
\hline \multicolumn{2}{|l}{${ }^{*} \mathrm{p}<0.05,{ }^{* *} p<0.01,{ }^{* * *} \mathrm{p}<0.001$ indicates statistical significance the difference between sex } & \\
\hline
\end{tabular}

Table 1: Descriptive Statistics

Weight change results show that $94.7 \%$ of the participants included in this study lost weight, while $79.2 \%$ experienced clinically-significant weight loss. The average $\% \mathrm{WL}$ was $7.8(\mathrm{SD}=7.5)$, which translated into an average loss of 7.1 kilograms $(\mathrm{SD}=6.4 \mathrm{~kg})$. BMI declined by 2.6 $(\mathrm{SD}=2.3)$, on average. Additionally, men saw 1.1 more \%WL than women $(\mathrm{t}=2.23, \mathrm{p}=026)$ and experienced a .44 more reduction in BMI following the BLE:BC program ( $\mathrm{t}=2.93, \mathrm{p}=0.003)$.

Based on results from questions in the ending survey, over $88 \%$ of participants expressed satisfaction with the BLE:BC program $(71.5 \%$ of the sample reported that they were very satisfied with the BLE:BC program and $17.1 \%$ reported being satisfied with the program). Following completion of the program, $90.4 \%$ indicated that healthy eating had become easier, while $7.7 \%$ indicated that healthy eating had become harder. In terms of program adherence, the results indicate a high level of adherence: only $8.6 \%$ reported giving "some effort," $35.0 \%$ gave "a lot of effort," and 56.0\% "gave it their all." For degree of participation in the program, $8.6 \%$ participated some, $24.3 \%$ participated a lot and $64.5 \%$ participated continuously from the beginning of the program.

\section{Baseline weight status and weight loss}

[Figures 1] shows \%WL by baseline weight status. Results of a Spearman correlation test show that baseline weight status is significantly associated with \%WL $(\mathrm{rs}=.12, \mathrm{p}<0.001)$. Compared to participants who reported normal weight at baseline, those who were overweight when they began the program experienced 3.4 greater $\% \mathrm{WL}$, those with class 1 obesity experienced 4.1 greater $\% \mathrm{WL}$, those with class 2 obesity experienced 4.1 greater $\% \mathrm{WL}$, and those with class 3 obesity experienced 4.7 greater \%WL. Figure 2 reports the association between baseline weight status and BMI change. Spearman correlation tests show that baseline weight status is significantly associated with change in BMI $(\mathrm{rs}=.45, \mathrm{p}<0.001)$. Participants with greater $\mathrm{BMI}$ at baseline experienced greater reductions in $\mathrm{BMI}$ during the BLE:BC program. Moreover, among those who began the program with any obesity, $66.5 \%$ achieved obesity remission and $2.2 \%$ achieved normal weight status.

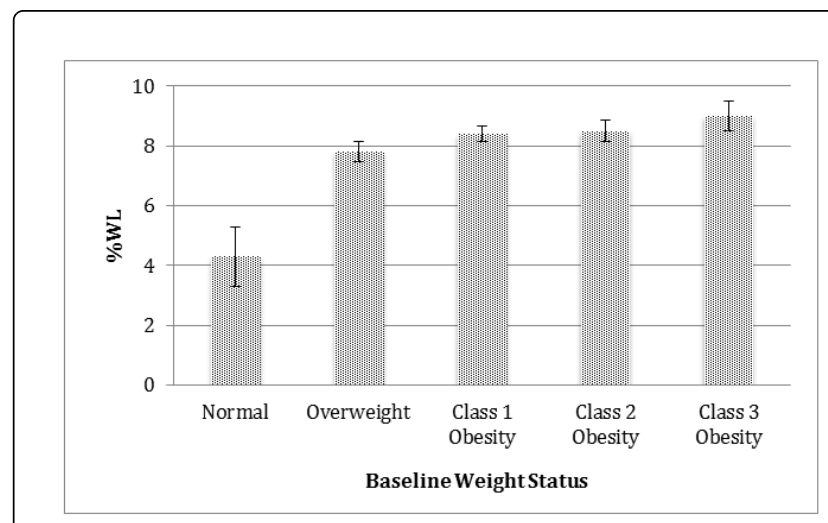

*Error bars represent $95 \%$ confidence intervals.

Figure 1: \%WL by Baseline Weight Status

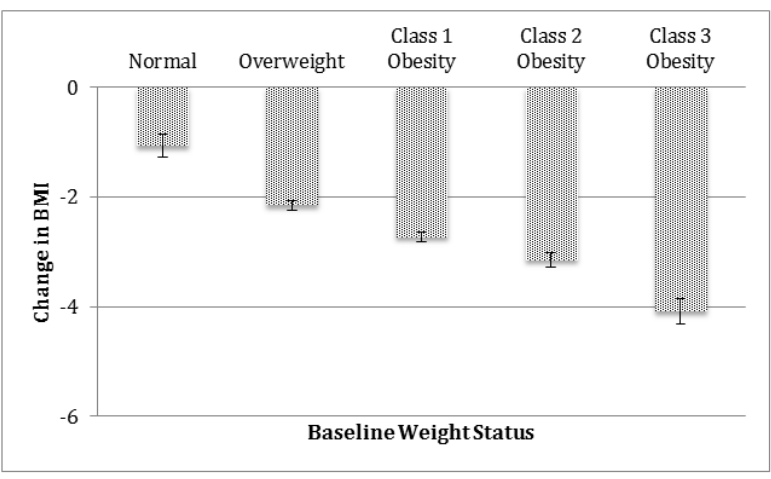

Figure 2: BMI Change by Baseline Weight Status

\section{Program adherence and weight loss}

To test the effect of program adherence on weight loss, we used Spearman correlation tests to examine the association of (a) level of effort and (b) program participation with two outcomes: \%WL and BMI change. As [Figures 3,4] show, \%WL increased significantly as participants' self-reported effort $(\mathrm{rs}=0.39, \mathrm{p}<0.001)$ and level of BLE: $B C$ program participation $(r s=0.34, p<0.001)$ rose. Similarly, participants who reported high levels of effort $(\mathrm{rs}=0.36, \mathrm{p}<0.001)$ and participation $(\mathrm{rs}=0.33, \mathrm{p}<0.001)$ experienced greater $\mathrm{BMI}$ reductions. 
Citation: Guan W, Thaw A, Grondhuis S, Schaechter A (2018) Evaluation of a Commercial Telehealth Weight Loss and Management Program. J Nutr Weight Loss 3: 1000114.

Page 6 of 8

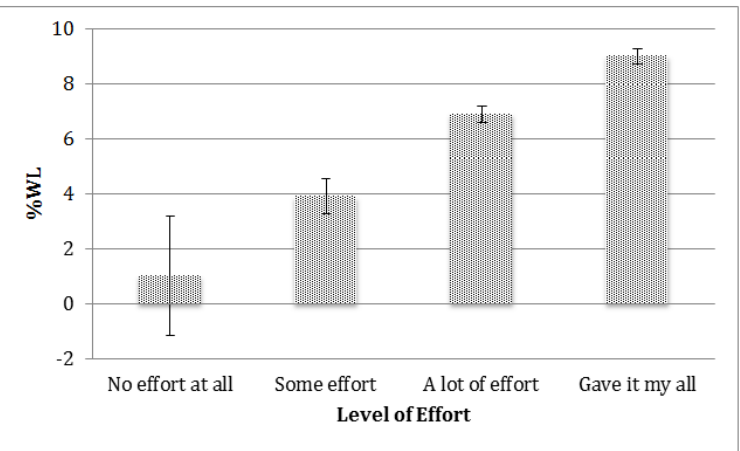

Figure 3: \%WL by Level of Effort

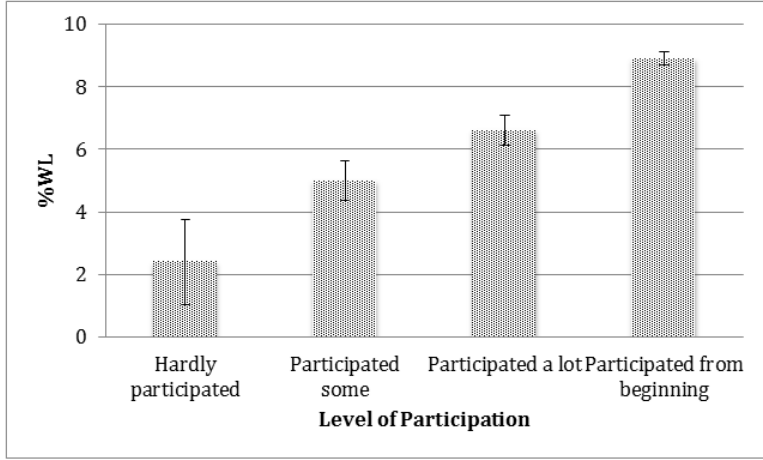

Figure 4: \%WL by Level of Participation

\section{Program satisfaction, healthy eating, and weight loss}

Chi-square tests show that clinically significant weight loss and satisfaction with the program was significantly associated (chi2=958.01, p <0.000). Shown in [Figure 5], participants who experienced clinically-significant weight loss were twice as likely to report being "very satisfied" $(79.3 \%)$ with the BLE:BC program than participants who either experienced less than 5\%WL (42.7\%) or did not lose weight at all (33.1\%). Similarly, chi-square tests show that clinically significant weight loss is also significantly associated with the likelihood of participants reporting that healthy eating became easier (chi2=758.4, $\mathrm{p}=0.000$ ). [Figure 6] shows that participants who experienced clinically-significant weight loss were more likely report that healthy eating became easier $(95.3 \%)$ than were participants who experienced less than $5 \%$ weight loss $(38.0 \%)$ or who did not lose any weight $(16.8 \%)$.

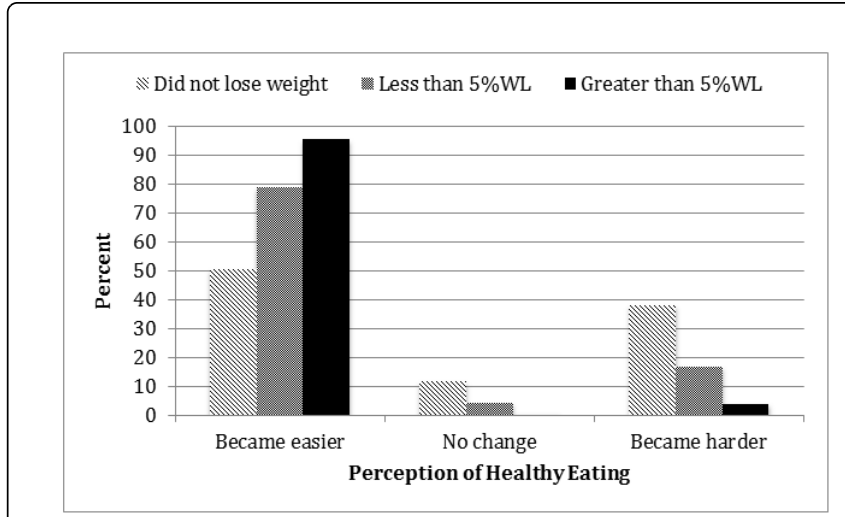

Figure 5: Program Satisfaction by Clinically-Significant Weight Loss

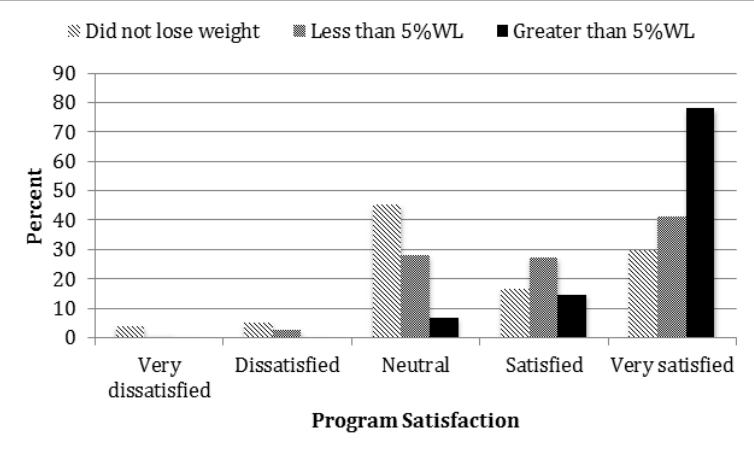

Figure 6: Healthy Eating by Clinically-Significant Weight Loss

\section{Discussion}

The purpose of the current study was to evaluate the efficacy of the BLE: BC program. The results of this study show support for the BLE: $\mathrm{BC}$ program as an effective telehealth (web-based) weight loss program. We found notable changes in body weight, BMI, and other behavioral measures. The majority of participants experienced clinically-significant weight loss. The most dramatic weight loss and BMI reduction occurred among participants who (a) were obese at baseline or (b) had high program adherence. Furthermore, satisfaction with the BLE: BC program was high, particularly among participants who experienced significant weight loss. The majority of participants also reported that healthy eating became easier following the program.

These results have important implications as it contributes to the limited existing evidence base for the efficacy of commercial weight loss programs. First, this study provides case series weight loss outcomes for a commercial telehealth weight loss program. According to a review of the literature, results for only four commercial telehealth weight loss programs have been published. This is due in part because of the preference for randomized control trials over observational studies. Randomized controlled trials are difficult to complete for commercial programs. Although randomized control trials remain the gold standard for evaluating program efficacy, they suffer from such 
limitations as (1) expense, (2) small samples, and (3) lack of generalizability to real-life population health.

Second, this study shows that, compared to prior studies of commercial web-based programs, the BLC: BC program produces substantial weight loss. Prior studies of similar programs such as eDiets.com, Lose It!, and the Biggest Loser Club showed modest weight loss results that ranged from $0.9 \% \mathrm{WL}$ to $3.3 \% \mathrm{WL}$ and $1.2 \mathrm{BMI}$ reduction. The BLE: $\mathrm{BC}$ 's average \%WL of $7.8 \%$ stands dramatically higher. Further, this exceeds $5 \% \mathrm{WL}$, meeting the threshold of clinically-significant weight loss. This is critically important, given that research has shown that weight loss between $5-10 \%$ is strongly associated with improvements in biomarkers for type 2 diabetes and cardiovascular disease [23-24].

Finally, the BLE: BC program emphasizes a significant change in nutritional intake that is grounded in the food addiction framework. Because the results show that almost $95 \%$ of reporting BLE: BC research participants lost weight and almost $80 \%$ experienced clinically-significant weight loss, the current study presents strong support for the design of the BLE:BC nutrition plan. The finding that weight loss rose significantly among those with higher program adherence provides additional support for the design of the program. However, future research will benefit from more in-depth evaluation of BLE: BC program adherence such as participants' intake of sugar or flour, two primary components of BLE.

\section{Conclusion}

Given the emergence of commercial telehealth (web-based) weight loss programs, it becomes important to evaluate scientifically the effectiveness of each program. BLE offers a strict but simple weight loss plan that can create substantial weight loss. Data concerning the longterm sustainability of weight loss among BLE participants need to be assessed to confirm the program's utility as a sustainable weight loss option. We note that BLE offers the only commercial telehealth weight loss program that shows the capacity to, on average, provide clinicallysignificant weight loss results.

\section{Limitations}

The observational case series research design of this study may introduce unintended bias, which may result from the lack of a control group, selection bias, and/or missing data. The present study uses a convenience sample that may suffer from selection bias. Participants who elected to participate in the BLE:BC research program and complete the surveys used in this study may be more likely to have lost more weight or be more motivated to lose weight. However, previous research has shown that, because estimates gathered in observational studies often approximate closely those of randomized, controlled trials, researchers may overstate bias in observational studies [25]. Additionally, the homogeneity of the sample--which may result in part from the cost of the BLE program may introduce an additional avenue for selection bias.

\section{References}

1. Khaodhiar L, McCowen KC, Blackburn GL (1999) Obesity and its comorbid conditions. Clin Cornerstone 2: 17-31.

2. Flegal KM, Kit BK, Orpana H, Graubard BI (2013) Association of allcause mortality with overweight and obesity using standard body mass index categories: a systematic review and meta-analysis Jama 309: 71-82.
3. Cawley J, Meyerhoefer C (2012) The medical care costs of obesity: an instrumental variables approach. J Health Econ 31: 219-230.

4. Cawley J, Rizzo JA, Haas K (2007) Occupation-specific absenteeism costs associated with obesity and morbid obesity. J Occup Environ Med 49: 1317-1324.

5. Gates DM, Succop P, Brehm BJ, Gillespie GL, Sommers BD (2008) Obesity and presenteeism: the impact of body mass index on workplace productivity. J Occup Environ Med 50: 39-45.

6. Neve M, Morgan PJ, Jones PR, Collins CE (2010) Effectiveness of webbased interventions in achieving weight loss and weight loss maintenance in overweight and obese adults: a systematic review with meta-analysis. Obes Rev 11: 306-321.

7. Tsai AG, Wadden TA (2005) Systematic review: an evaluation of major commercial weight loss programs in the United States. Ann Intern Med 142: 56-66.

8. Womble, LG, Wadden TA, McGuckin BG, Sargent SL, Rothman RA, Krauthamer EES (2004) A randomized controlled trial of a commercial internet weight loss program. Obes Res 12: 1011-1018.

9. Gudzune KA, Doshi RS, Mehta AK, Chaudhry ZW, Jacobs DK et al. (2015) Efficacy of commercial weight-loss programs: an updated systematic review. Ann Intern Med 162: 501-512.

10. Allen JK, Stephens J, Dennison HCR, Stewart KJ, Hauck S (2013) Randomized controlled pilot study testing use of smartphone technology for obesity treatment. J Obes 2013.

11. Collins CE, Morgan PJ, Jones P, Fletcher K, Martin J et al. (2012) A 12week commercial web-based weight-loss program for overweight and obese adults: randomized controlled trial comparing basic versus enhanced features. JMIR, 14(2): e57.

12. Ifland J, Preuss HG, Marcus MT, Rourke KM, Taylor W et al. (2015) Clearing the confusion around processed food addiction. J Am Coll Nutr. 34: $240-243$.

13. Volkow ND, Wise RA, Baler R (2017) The dopamine motive system: implications for drug and food addiction. Nat Rev Neurosci 18: 741-752.

14. Benton D, Young HA (2016) A meta-analysis of the relationship between brain dopamine receptors and obesity: a matter of changes in behavior rather than food addiction?. Int J Obes 40 :S12-21.

15. Yeh J, Trang A, Henning SM, Wilhalme H, Carpenter C et al.(2016) Food cravings, food addiction, and a dopamine-resistant (DRD2 A1) receptor polymorphism in Asian American college students. Asia Pac J Clin Nutr 25: 424-429.

16. Gordon EL, Ariel DAH, Bauman V, Merlo LJ (2018) What Is the Evidence for "Food Addiction?" A Systematic Review. Nutrients 10: 477.

17. Volkow ND, Wang GJ, Baler RD (2011) Reward, dopamine and the control of food intake: implications for obesity. Trends Cogn Sci 15: 37-46.

18. Avena NM, Rada P, Hoebel BG (2008) Evidence for sugar addiction: behavioral and neurochemical effects of intermittent, excessive sugar intake. Neurosci Biobehav Rev 32: 20-39.

19. Avena NM, Bocarsly ME, Rada P, Kim A, Hoebel BG (2008) After daily bingeing on a sucrose solution, food deprivation induces anxiety and accumbens dopamine/acetylcholine imbalance. Physiol Behav 94: 309-315.

20. MacDonald IA (2016) A review of recent evidence relating to sugars, insulin resistance and diabetes. Eur J Nutr 55: 17-23.

21. Chandon P, Wansink B (2007) Is obesity caused by calorie underestimation? A psychophysical model of meal size estimation. J Mark Res 44: 84-99.

22. Lichtman SW, Pisarska K, Berman ER, Pestone M, Dowling $\mathrm{H}$ et al. (1992) Discrepancy between self-reported and actual caloric intake and exercise in obese subjects. N Engl J Med 327: 1893-1898.

23. Vidal J (2002) Updated review on the benefits of weight loss. Int J Obes 26: S25-S28.

24. Van GLF, Mertens IL, Ballaux D (2005) What is the relationship between risk factor reduction and degree of weight loss?. Eur Heart J Suppl 7: L21L26. 
Citation: Guan W, Thaw A, Grondhuis S, Schaechter A (2018) Evaluation of a Commercial Telehealth Weight Loss and Management Program. J Nutr Weight Loss 3: 1000114.

Page 8 of 8

25. Concato J, Shah N,Horwitz R I (2000). Randomized, controlled trials, observational studies, and the hierarchy of research designs. N Engl J Med 342: 1887-1892. 